\title{
Multivariable Super Twisting Based Robust Trajectory Tracking Control for Small Unmanned Helicopter
}

\author{
Xing Fang, Aiguo Wu, Yujia Shang, and Chunyan Du \\ School of Electrical Engineering and Automation, Tianjin University, No. 92, Weijin Road, Nankai District, Tianjin 300072, China \\ Correspondence should be addressed to Xing Fang; xingf@tju.edu.cn
}

Received 18 September 2014; Revised 23 February 2015; Accepted 21 March 2015

Academic Editor: Alain Vande Wouwer

Copyright (C) 2015 Xing Fang et al. This is an open access article distributed under the Creative Commons Attribution License, which permits unrestricted use, distribution, and reproduction in any medium, provided the original work is properly cited.

\begin{abstract}
This paper presents a highly robust trajectory tracking controller for small unmanned helicopter with model uncertainties and external disturbances. First, a simplified dynamic model is developed, where the model uncertainties and external disturbances are treated as compounded disturbances. Then the system is divided into three interconnected subsystems: altitude subsystem, yaw subsystem, and horizontal subsystem. Second, a disturbance observer based controller (DOBC) is designed based upon backstepping and multivariable super twisting control algorithm to obtain robust trajectory tracking property. A sliding mode observer works as an estimator of the compounded disturbances. In order to lessen calculative burden, a first-order exact differentiator is employed to estimate the time derivative of the virtual control. Moreover, proof of the stability of the closed-loop system based on Lyapunov method is given. Finally, simulation results are presented to illustrate the effectiveness and robustness of the proposed flight control scheme.
\end{abstract}

\section{Introduction}

The helicopters have many advantages over ordinary fixedwing vehicles. With the capability of hovering, vertical taking-off and landing, high levels of agility, and maneuverability, unmanned helicopters have been used in urban and mountainous environment. They have wide application prospects in the field of military and civilian. However, the unmanned helicopter system is a nonlinear, underactuated, and strong coupled system. Consequently, it is a challenging research area to design high-performance controllers $[1,2]$.

Generally, control methods for unmanned helicopters can be classified into two categories: linear controllers and nonlinear controllers. Traditional methods are based on linear model, such as PID [3], LQR [4], $H_{\infty}[5,6]$, and $\mu$ synthesis [7]. Nevertheless these linear control methods are only effective when the states of the unmanned helicopter system are near the equilibrium points. In order to overcome these shortcomings, many nonlinear control methods are developed, such as feedback linearization [8], model predictive control [9], intelligent control [10], backstepping control [11-14], and sliding mode control [15-19]. Among these methods, backstepping control algorithm has drawn much attention of many researchers. Backstepping designs can avoid cancellation of useful nonlinearities and often introduce additional nonlinear terms to improve transient performance. Moreover, backstepping is an effective control algorithm for underactuated systems as unmanned helicopter system [13]. The key idea behind the backstepping design technique is that some virtual control inputs are introduced to counterbalance the number of inputs and outputs. However, exact knowledge of the plant model is necessary for backstepping control design. It is practically impossible to obtain the exact model of unmanned helicopter system. Sliding mode control algorithm is known as a robust control method [15, 16]. It is able to make the system keep robustness and stability even in the presence of disturbances. Among all of the sliding mode control algorithms, super twisting control algorithm is the most popular one [17-19]. This algorithm possesses the property of finite time convergence. And it can alleviate chattering phenomena, because the switching control part is hidden into the derivative of the sliding mode variable [20]. 
However, traditional super twisting control algorithm only keeps robustness with matched disturbances and only can deal with single-input single-output systems.

The combination of backstepping and multivariable super twisting control algorithms makes it possible to control the unmanned helicopters with the high-order, multiple-input multiple-output, underactuated, and disturbance rejection problem effectively. A sliding mode observer is developed to estimate the compounded disturbances including matched and unmatched disturbances [21]. By integrating the observer output into the controller, the compounded disturbances can be compensated for to improve the robust performance. Thus, this disturbance observer based control (DOBC) scheme will be robust with both matched and unmatched disturbances. Furthermore, a first-order exact differentiator is employed to estimate the time derivative of the virtual control, which can avoid large calculation load [22]. It is proved by Lyapunov technique that the closed-loop tracking error is globally asymptotically stable.

The organization of this paper is as follows. Section 2 briefly recalls the full dynamic model of small unmanned helicopters with compounded disturbances. In Section 3, the complete design procedure of the disturbance observer based controller (DOBC) is presented. The stability analysis of the proposed controller is carried out in Section 4. In Section 5, numerical simulations are conducted to demonstrate the robust performance of the proposed control method. Section 6 presents the conclusion.

\section{Helicopter Model}

The small unmanned helicopter is a highly nonlinear, strong coupled, and underactuated system with model uncertainties and external disturbances [2]. It is difficult to obtain a complete model for its high order and large number of parameters. Moreover, it is unmanageable for us to design a practical controller. Therefore, this paper treats the unmanned helicopter as a six-degrees-of-freedom rigid-body model with simplified force and moment generation process and considers model uncertainties and external disturbances as compounded disturbances [9]. Thus, we will proceed on the flight control design based on the simplified unmanned helicopter model.

The first step to the development of the rigid-body equations of motion is the definition of two reference frames. The first one is the inertial coordinate defined as $N=$ $\left\{O_{n}, x_{n}, y_{n}, z_{n}\right\}$. The second is the body fixed reference frame defined as $B=\left\{O_{b}, x_{b}, y_{b}, z_{b}\right\}$. The small unmanned helicopter system can be seen in Figure 1.

The rigid-body dynamics of the unmanned helicopter can be expressed as follows:

$$
\begin{aligned}
& \dot{P}=V, \\
& \dot{V}=g e_{3}+\frac{1}{m} R(\Theta)\left(f+f_{d V}\right), \\
& \dot{\Theta}=\Pi(\Theta) \Omega, \\
& \dot{\Omega}=-J^{-1} \Omega \times J \Omega+J^{-1}\left(\tau+\tau_{d \Omega}\right),
\end{aligned}
$$

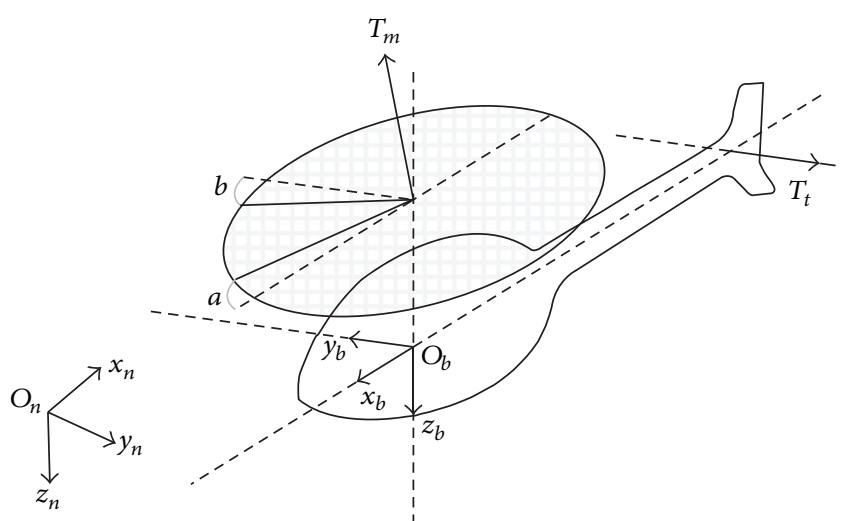

FIGURE 1: Small unmanned helicopter system model schematic diagram.

where $g$ is the acceleration due to gravity, $m$ is the total mass of the unmanned helicopter, $J=\operatorname{diag}\left(J_{x x}, J_{y y}, J_{z z}\right)$ is the diagonal inertia matrix, and $e_{3}=\left[\begin{array}{lll}0 & 0 & 1\end{array}\right]^{T} \cdot P=\left[\begin{array}{lll}x & y & z\end{array}\right]^{T}$ and $V=\left[\begin{array}{lll}u & v & w\end{array}\right]^{T}$ represent the position and velocity in the inertial frame, and $\Theta=\left[\begin{array}{lll}\phi & \theta & \psi\end{array}\right]^{T}$ and $\Omega=\left[\begin{array}{lll}p & q & r\end{array}\right]^{T}$ represent the Euler angles and angular rates in the body frame. The rotation matrix $R(\Theta)$ and the attitude kinematic matrix $\Pi(\Theta)$ are defined, respectively, as follows:

$$
\begin{aligned}
R(\Theta) & =\left[\begin{array}{ccc}
C_{\theta} C_{\psi} & S_{\phi} S_{\theta} C_{\psi}-C_{\phi} S_{\psi} & C_{\phi} S_{\theta} C_{\psi}+S_{\phi} S_{\psi} \\
C_{\theta} S_{\psi} & S_{\phi} S_{\theta} S_{\psi}+C_{\phi} C_{\psi} & C_{\phi} S_{\theta} S_{\psi}-S_{\phi} C_{\psi} \\
-S_{\theta} & S_{\phi} C_{\theta} & C_{\phi} C_{\theta}
\end{array}\right] \\
\Pi(\Theta) & =\left[\begin{array}{ccc}
1 & S_{\phi} T_{\theta} & C_{\phi} T_{\theta} \\
0 & C_{\phi} & -S_{\phi} \\
0 & \frac{S_{\phi}}{C_{\theta}} & \frac{C_{\phi}}{C_{\theta}}
\end{array}\right],
\end{aligned}
$$

where the compact notation $C$ denotes $\cos (\cdot), S$ denotes $\sin (\cdot)$, and $T$ denotes $\tan (\cdot)$.

$f$ and $\tau$ are the external force and moment vectors, generated by the main rotor thrust and the tail rotor thrust, which are the main powers of the unmanned helicopter. $f_{d V}$ and $\tau_{d \Omega}$ are normalized force and moment disturbances that include internal couplings, unmodelled dynamics, and wind gusts. They are defined, respectively, as

$$
\begin{aligned}
f & =\left[\begin{array}{c}
0 \\
0 \\
T_{m}
\end{array}\right] \\
\tau & =J\left[\begin{array}{c}
L_{a} a+L_{b} b \\
M_{a} a+M_{b} b \\
N_{r} r+N_{\mathrm{col}} \delta_{\mathrm{col}}+N_{\mathrm{ped}} \delta_{\mathrm{ped}}
\end{array}\right] \\
f_{d V} & =m\left[\begin{array}{lll}
f_{d u} & f_{d v} & f_{d w}
\end{array}\right]^{T} \\
\tau_{d \Omega} & =J\left[\begin{array}{lll}
\tau_{d p} & \tau_{d q} & \tau_{d r}
\end{array}\right]^{T},
\end{aligned}
$$


where $T_{m}$ is main rotor thrust controlled by collective pitch $\delta_{\mathrm{col}}: T_{m} \approx m\left(-g+Z_{w} w+Z_{\mathrm{col}} \delta_{\mathrm{col}}\right)$, and $\delta_{\text {ped }}$ is the collective pitch of the tail rotor. The parameters $L_{a}, L_{b}, M_{a}, M_{b}, N_{r}, N_{\text {col }}, N_{\text {ped }}$ can be obtained by system identification. The longitudinal flapping angle and lateral flapping angle $a, b$ cannot be measured directly, but they are controlled by lateral and longitudinal cyclic $\delta_{\text {lat }}, \delta_{\text {lon }}$ as follows:

$$
\begin{aligned}
& a=-\tau_{m} q+A_{\text {lat }} \delta_{\text {lat }}+A_{\text {lon }} \delta_{\text {lon }} \\
& b=-\tau_{m} p+B_{\text {lat }} \delta_{\text {lat }}+B_{\text {lon }} \delta_{\text {lon }},
\end{aligned}
$$

where the parameters $\tau_{m}, A_{\text {lat }}, A_{\text {lon }}, B_{\text {lat }}, B_{\text {lon }}$ also can be obtained by system identification.

Substituting (8) into (5), a more compact form of the torque can be obtained as follows:

$$
\tau=J(A \Omega+B u),
$$

where

$$
\begin{aligned}
A & =\left[\begin{array}{ccc}
-\tau_{m} L_{b} & -\tau_{m} L_{a} & 0 \\
-\tau_{m} M_{b} & -\tau_{m} M_{a} & 0 \\
0 & 0 & N_{r}
\end{array}\right] \\
B & =\left[\begin{array}{cccc}
0 & L_{\text {lon }} & L_{\text {lat }} & 0 \\
0 & M_{\text {lon }} & M_{\text {lat }} & 0 \\
N_{\text {col }} & 0 & 0 & N_{\text {ped }}
\end{array}\right] .
\end{aligned}
$$

The parameters in these matrices can be given as follows:

$$
\begin{gathered}
L_{\text {lon }}=L_{a} A_{\text {lon }}+L_{b} B_{\text {lon }} \\
L_{\text {lat }}=L_{a} A_{\text {lat }}+L_{b} B_{\text {lat }} \\
M_{\text {lon }}=M_{a} A_{\text {lon }}+M_{b} B_{\text {lon }} \\
M_{\text {lat }}=M_{a} A_{\text {lat }}+M_{b} B_{\text {lat }} .
\end{gathered}
$$

The modified unmanned helicopter model combining (1)-(11) can be described in the following form:

$$
\begin{aligned}
& \dot{P}=V \\
& \dot{V}=g e_{3}+R(\Theta) e_{3}\left(-g+Z_{w} w+Z_{\mathrm{col}} \delta_{\mathrm{col}}\right)+d_{V} \\
& \dot{\Theta}=\Pi(\Theta) \Omega \\
& \dot{\Omega}=-J^{-1} \Omega \times J \Omega+A \Omega+B u+d_{\Omega},
\end{aligned}
$$

where $x=\left[\begin{array}{llll}P^{T} & V^{T} & \Theta^{T} & \Omega^{T}\end{array}\right]^{T}$ is the helicopter state vector, $u=\left[\begin{array}{llll}\delta_{\text {col }} & \delta_{\text {lon }} & \delta_{\text {lat }} & \delta_{\text {ped }}\end{array}\right]^{T}$ is the control input vector, and $d_{V}=\left[\begin{array}{lll}d_{u} & d_{v} & d_{w}\end{array}\right]^{T}=R(\Theta) f_{d V} / m$ and $d_{\Omega}=\left[\begin{array}{lll}d_{p} & d_{q} & d_{r}\end{array}\right]^{T}=$ $J^{-1} \tau_{d \Omega}$ are the compounded disturbances vector acting on the unmanned helicopter.

For convenience of controller design, the following formula will be used:

$$
\dot{R}(\Theta)=R(\Theta) S(\Omega),
$$

where $S(\Omega)$ is a skew symmetric matrix, and it can be defined as follows:

$$
S(\Omega)=\left[\begin{array}{ccc}
0 & -r & q \\
r & 0 & -p \\
-q & p & 0
\end{array}\right]
$$

Actually, (16) has the same meaning as (14).

According to the feature of the unmanned helicopter model, the system can be divided into three interconnected subsystems: altitude subsystem, yaw subsystem, and horizontal subsystem. The controller for each subsystem can be designed separately. Consider

$$
S_{1}:\left\{\begin{array}{l}
\dot{z}=w \\
\dot{w}=g+r_{33}\left(-g+Z_{w} w+Z_{\mathrm{col}} \delta_{\mathrm{col}}\right)+d_{w}
\end{array}\right.
$$

$S_{2}$ :

$$
\left\{\begin{array}{l}
\dot{\psi}=\frac{S_{\phi}}{C_{\theta}} q+\frac{C_{\phi}}{C_{\theta}} r \\
\dot{r}=-\frac{I_{y y}-I_{x x}}{I_{z z}} p q+N_{r} r+N_{\mathrm{col}} \delta_{\mathrm{col}}+N_{\mathrm{ped}} \delta_{\mathrm{ped}}+d_{r}
\end{array}\right.
$$

$S_{3}:\left\{\begin{array}{l}\dot{P}_{1}=V_{1} \\ \dot{V}_{1}=R_{1}\left(-g+Z_{w} w+Z_{\mathrm{col}} \delta_{\mathrm{col}}\right)+d_{V 1} \\ \dot{R}_{1}=\Pi_{1} \Omega_{1} \\ \dot{\Omega}_{1}=f_{1}\left(\Omega_{1}\right)+A_{1} \Omega_{1}+B_{1} u_{1}+d_{\Omega 1},\end{array}\right.$

where $P_{1}=\left[\begin{array}{ll}x & y\end{array}\right]^{T}, V_{1}=\left[\begin{array}{ll}u & v\end{array}\right]^{T}, R_{1}=\left[\begin{array}{ll}r_{13} & r_{23}\end{array}\right]^{T}, \Omega_{1}=$ $\left[\begin{array}{ll}p & q\end{array}\right]^{T}, u_{1}=\left[\begin{array}{ll}\delta_{\text {lon }} & \delta_{\text {lat }}\end{array}\right]^{T}, d_{V 1}=\left[\begin{array}{ll}d_{u} & d_{v}\end{array}\right]^{T}, d_{\Omega 1}=\left[\begin{array}{ll}d_{p} & d_{q}\end{array}\right]^{T}$, $\Pi_{1}=\left[\begin{array}{ll}-r_{12} & r_{11} \\ -r_{22} & r_{21}\end{array}\right], f_{1}\left(\Omega_{1}\right)=\left[-\left(\left(I_{z z}-I_{y y}\right) / I_{x x}\right) q r-\left(\left(I_{x x}-\right.\right.\right.$ $\left.\left.\left.I_{z z}\right) / I_{y y}\right) p r\right]^{T}, A_{1}=\left[\begin{array}{cc}-\tau_{m} L_{b} & -\tau_{m} L_{a} \\ -\tau_{m} M_{b} & -\tau_{m} M_{a}\end{array}\right]$, and $B_{1}=\left[\begin{array}{cc}L_{\text {lon }} & L_{\text {lat }} \\ M_{\text {lon }} & M_{\text {lat }}\end{array}\right]$, and $r_{i, j}$ represents the element of $i$ th row and $j$ th column of the rotation matrix $R(\Theta)$.

\section{Controller Design for Small Unmanned Helicopter}

3.1. Modified Multivariable Super Twisting Algorithm. Here, a modified multivariable super twisting algorithm is used for our controller design. In order to make the multivariable super twisting algorithm [23] more suitable for the small unmanned helicopter, some modifications are made as follows.

Consider the multivariable system

$$
\begin{aligned}
& \dot{x}_{1}=-\lambda \frac{x_{1}}{\left\|x_{1}\right\|^{1 / 2}}+x_{2}+\rho_{1}\left(t, x_{1}\right) \\
& \dot{x}_{2}=-\alpha \frac{x_{1}}{\left\|x_{1}\right\|}+\rho_{2}(t),
\end{aligned}
$$

where $x_{1}, x_{2} \in R^{m}, \lambda$, and $\alpha$ are positive constants and $\rho_{1}\left(t, x_{1}\right), \rho_{2}(t)$ are perturbations. 
Assumption 1. The perturbations $\rho_{1}\left(t, x_{1}\right)$ and $\rho_{2}(t)$ are globally bounded by

$$
\begin{aligned}
& \left\|\rho_{1}\right\| \leq \delta_{1}\left\|x_{1}\right\|^{1 / 2} \\
& \left\|\rho_{2}\right\| \leq \delta_{2}
\end{aligned}
$$

for some constants $\delta_{1}, \delta_{2}>0$.

Theorem 2. Under Assumption 1, if the parameters $\lambda$ and $\alpha$ satisfy

$$
\begin{aligned}
& \lambda>\max \left\{2 \delta_{1}, \frac{1}{2} \delta_{1}+\sqrt{\frac{1}{4} \delta_{1}^{2}+2 \delta_{2}}\right\} \\
& \alpha>\frac{2 \delta_{1} \lambda^{3}+\left(1.125 \delta_{1}^{2}+3 \delta_{2}\right) \lambda^{2}+3 \delta_{1} \delta_{2} \lambda+2 \delta_{2}^{2}}{\lambda\left(\lambda-2 \delta_{1}\right)}
\end{aligned}
$$

all the trajectories of system (21) will converge to origin in finite time.

Analysis and Proof. The multivariable super twisting algorithm in Theorem 2 is developed based on the proposal in [23]. Some modifications are made to this algorithm. First, it is assumed that the perturbation $\rho_{1}\left(t, x_{1}\right)$ satisfies $\left\|\rho_{1}\right\| \leq$ $\delta_{1}\left\|x_{1}\right\|^{1 / 2}$ instead of $\left\|\rho_{1}\right\| \leq \delta_{1}\left\|x_{1}\right\|$ in [23]. Second, the linear control terms are removed in multivariable system (21).

The stability analyses of these two algorithms are similar. And the details of the proof can be found in article [23]. So we only give the outline of the stability analysis below.

We define a Lyapunov function candidate for system (21) as follows:

$$
\begin{aligned}
& V\left(x_{1}, x_{2}\right) \\
& =2 \alpha\left\|x_{1}\right\|+\frac{1}{2} x_{2}^{T} x_{2} \\
& \quad+\frac{1}{2}\left(\lambda \frac{x_{1}}{\left\|x_{1}\right\|^{1 / 2}}-x_{2}\right)^{T}\left(\lambda \frac{x_{1}}{\left\|x_{1}\right\|^{1 / 2}}-x_{2}\right) .
\end{aligned}
$$

Define a vector $\varsigma=\operatorname{col}\left(\left\|x_{1}\right\|^{1 / 2},\left\|x_{2}\right\|\right)$. Considering system (21) and perturbations (22), the time derivative of (24) can be obtained as follows:

$$
\dot{V} \leq-\frac{1}{\left\|x_{1}\right\|^{1 / 2}} \varsigma^{T} Q \varsigma
$$

where

$$
Q=\left[\begin{array}{cc}
\lambda \alpha+\frac{\lambda^{3}}{2}-\left(2 \alpha+\frac{\lambda^{2}}{2}\right) \delta_{1}-\lambda \delta_{2} & * \\
-\left(\frac{\lambda^{2}}{2}+\frac{3}{4} \lambda \delta_{1}+\delta_{2}\right) & \frac{\lambda}{2}
\end{array}\right]
$$

If $Q>0$, then $\dot{V}<0$. It is easy to see that this is the case if the parameters satisfy

$$
\begin{aligned}
& \lambda>\max \left\{2 \delta_{1}, \frac{1}{2} \delta_{1}+\sqrt{\frac{1}{4} \delta_{1}^{2}+2 \delta_{2}}\right\} . \\
& \alpha>\frac{2 \delta_{1} \lambda^{3}+\left(1.125 \delta_{1}^{2}+3 \delta_{2}\right) \lambda^{2}+3 \delta_{1} \delta_{2} \lambda+2 \delta_{2}^{2}}{\lambda\left(\lambda-2 \delta_{1}\right)}
\end{aligned}
$$

Define $\xi=\operatorname{col}\left(x_{1} /\left\|x_{1}\right\|^{1 / 2}, x_{2}\right)$, and note that $\|\varsigma\|=\|\xi\|$ for all values of $x_{1}$ and $x_{2}$. So we can derive $V=\xi^{T} P \xi \leq \lambda_{\text {max }}(P)\|\xi\|^{2}$ and $\dot{V} \leq-\left(1 /\left\|x_{1}\right\|^{1 / 2}\right) \lambda_{\min }(Q)\|\xi\|^{2}$, where $P$ is an appropriate symmetric positive definite matrix. Finally, we can get that

$$
\dot{V} \leq-\gamma V^{1 / 2}
$$

where $\gamma=\lambda_{\text {min }}(Q) \sqrt{\lambda_{\text {min }}(P)} / \lambda_{\text {max }}(P)$.

Therefore, $\left(x_{1}(t), x_{2}(t)\right)$ will converge to $\left(x_{1}, x_{2}\right)=(0,0)$. And $\dot{x}_{1}(t)$ will converge to $\dot{x}_{1}=0$ in finite time, because the right hand of the first equation in (21) equals zero when $\left(x_{1}, x_{2}\right)=(0,0)$ and $\rho_{1}(t, 0)=0$.

Remark 3. The proposed multivariable super twisting algorithm is developed by modifying the algorithm in [23]. Now we will clarify the motivations for these modifications as follows. First, the starting points of these two papers are different. The article [23] is a purely theoretical research work, whose goal is to propose a new algorithm theoretically without considering the particular practical application problem. However, the fundamental purpose of our paper is to develop an appropriate algorithm to control the unmanned helicopters effectively. Thus, we need to modify the existing algorithm to make it applicable for unmanned helicopters. Second, in our helicopter control system, the actual meaning of $\rho_{1}\left(t, x_{1}\right)$ is the disturbance estimation error, which is a very small perturbation rather than a linearly growing one. Therefore, it will be reasonable to suppose that $\rho_{1}\left(t, x_{1}\right)$ satisfies $\left\|\rho_{1}\right\| \leq \delta_{1}\left\|x_{1}\right\|^{1 / 2}$ instead of $\left\|\rho_{1}\right\| \leq \delta_{1}\left\|x_{1}\right\|$ in [23]. Moreover, when $x_{1}$ is small $\left(\left\|x_{1}\right\|<1\right)$, we can derive $\delta_{1}\left\|x_{1}\right\|^{1 / 2}>\delta_{1}\left\|x_{1}\right\|$. Therefore, the proposed algorithm in our paper can tolerate stronger perturbations near the origin than the one in [23]. Third, since the perturbations $\rho_{1}\left(t, x_{1}\right)$ and $\rho_{2}(t)$ both are not linearly growing ones, it is not necessary to use the linear control terms in system (21), which deal with the linearly growing perturbations effectively $[18,23]$. The proposed algorithm is able to deal with the strong perturbations near the origin, such as $\rho_{1}\left(t, x_{1}\right)$ and $\rho_{2}(t)$ in this situation. And the modified algorithm needs fewer parameters than the one in [23]. Therefore, it is more convenient to be applied for the unmanned helicopter system. Moreover, the simulation results in Section 5 will support our analysis that the modified multivariable super twisting algorithm is very effective in controlling the unmanned helicopter.

3.2. Disturbance Observer Based Controller (DOBC) Design. In this section, a disturbance observer based controller (DOBC) is proposed for the unmanned helicopter system. The composite controller structure is illustrated in Figure 2. 


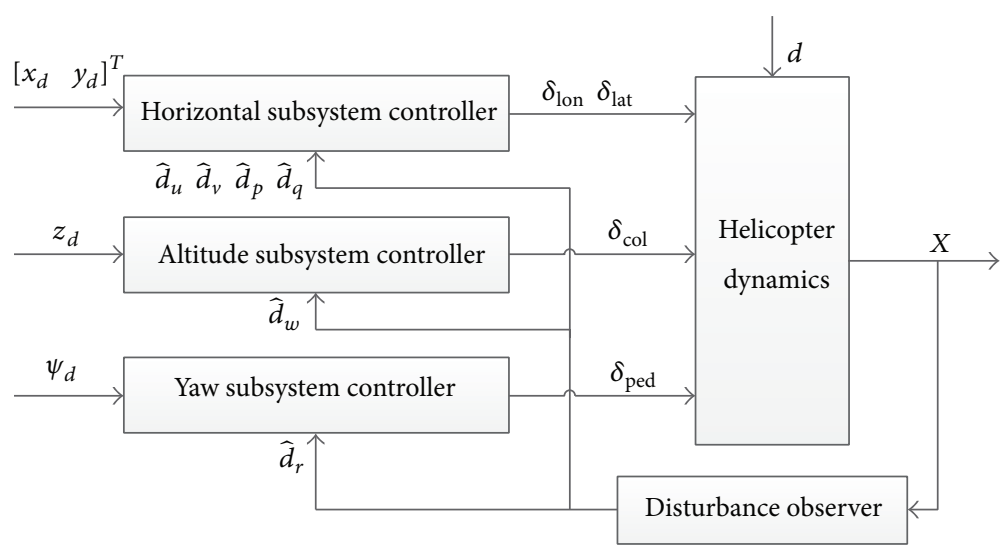

FIGURE 2: Controller structure.

The main control objective of this paper is to design the control inputs $u=\left[\begin{array}{llll}\delta_{\text {col }} & \delta_{\text {lon }} & \delta_{\text {lat }} & \delta_{\text {ped }}\end{array}\right]^{T}$ in order to track the reference trajectories of $\left[\begin{array}{lll}x_{d}(t) & y_{d}(t) & z_{d}(t)\end{array}\right]^{T}, \psi_{d}(t)$ and to stabilize the pitch and roll angles $\theta(t), \varphi(t)$ in the presence of compounded disturbances. To deal with the subsequent control development, some assumptions are made as follows.

Assumption 4. The reference trajectories are available. Moreover, $z_{d}(t)$ and $\psi_{d}(t)$ are $C^{2}$ functions of time, and $x_{d}(t)$ and $y_{d}(t)$ are $C^{4}$ functions of time.

Assumption 5. It is assumed that $|\theta(t)|<\pi / 2$ and $|\phi(t)|<\pi / 2$ for $t \geq 0$.

Obviously, this assumption can ensure the attitude kinematic matrix $\Pi(\Theta)$ defined in (3) will not be singularity [1214].

The detailed design process is described as follows.

3.2.1. Multivariable Super Twisting Based Observer. In the controller design procedure, the estimated disturbances $\widehat{d}_{V}=$ $\left[\begin{array}{lll}\widehat{d}_{u} & \widehat{d}_{v} & \widehat{d}_{w}\end{array}\right]^{T}$ and $\widehat{d}_{\Omega}=\left[\begin{array}{lll}\widehat{d}_{p} & \widehat{d}_{q} & \widehat{d}_{r}\end{array}\right]^{T}$ will be used, which are estimated by multivariable super twisting observers.

Considering the following dynamic models:

$$
\begin{aligned}
& \dot{V}=g e_{3}+R(\Theta) e_{3}\left(-g+Z_{w} w+Z_{\text {col }} \delta_{\text {col }}\right)+d_{V} \\
& \dot{\Omega}=-J^{-1} \Omega \times J \Omega+A \Omega+B u+d_{\Omega},
\end{aligned}
$$

the state-like observers are designed as follows:

$$
\begin{aligned}
& \dot{\hat{V}}=g e_{3}+R(\Theta) e_{3}\left(-g+Z_{w} w+Z_{\mathrm{col}} \delta_{\mathrm{col}}\right)+\widehat{d}_{V} \\
& \widehat{d}_{V}=\lambda_{V} \frac{e_{V}}{\left\|e_{V}\right\|^{1 / 2}}+z_{V} \\
& \dot{z}_{V}=\alpha_{V} \frac{e_{V}}{\left\|e_{V}\right\|},
\end{aligned}
$$

$$
\begin{aligned}
& \dot{\bar{\Omega}}=-J^{-1} \Omega \times J \Omega+A \Omega+B u+\widehat{d}_{\Omega} \\
& \widehat{d}_{\Omega}=\lambda_{\Omega} \frac{e_{\Omega}}{\left\|e_{\Omega}\right\|^{1 / 2}}+z_{\Omega} \\
& \dot{z}_{\Omega}=\alpha_{\Omega} \frac{e_{\Omega}}{\left\|e_{\Omega}\right\|},
\end{aligned}
$$

where $V$ and $\Omega$ are the outputs of sensors, regarding real values. $\widehat{V}$ and $\widehat{\Omega}$ are the estimated states of the observers.

State estimation errors can be defined as follows:

$$
\begin{aligned}
& e_{V}=V-\widehat{V} \\
& e_{\Omega}=\Omega-\widehat{\Omega} .
\end{aligned}
$$

Combining (29) and (31), we can derive

$$
\begin{aligned}
& \dot{e}_{V}=d_{V}-\widehat{d}_{V} \\
& \widehat{d}_{V}=\lambda_{V} \frac{e_{V}}{\left\|e_{V}\right\|^{1 / 2}}+z_{V} \\
& \dot{z}_{V}=\alpha_{V} \frac{e_{V}}{\left\|e_{V}\right\|} .
\end{aligned}
$$

Similarly, combining (30) and (32), we can derive

$$
\begin{aligned}
& \dot{e}_{\Omega}=d_{\Omega}-\widehat{d}_{\Omega} \\
& \widehat{d}_{\Omega}=\lambda_{\Omega} \frac{e_{\Omega}}{\left\|e_{\Omega}\right\|^{1 / 2}}+z_{\Omega} \\
& \dot{z}_{\Omega}=\alpha_{\Omega} \frac{e_{\Omega}}{\left\|e_{\Omega}\right\|} .
\end{aligned}
$$

Theorem 6. If the derivatives of disturbances are globally bounded by $\left\|\dot{d}_{i}\right\|<D_{i}, i=V, \Omega$, and the parameters in observers (31) and (32) satisfy

$$
\begin{aligned}
& \lambda_{i} \geq \sqrt{2 D_{i}}, \\
& \alpha_{i} \geq 3 D_{i}+\frac{2 D_{i}^{2}}{\lambda_{i}^{2}},
\end{aligned}
$$


the estimated disturbances $\widehat{d}_{i}$ converge to real disturbances $d_{i}$ in finite time.

Proof. The observer dynamics (34) and (35) can be rewritten as follows:

$$
\begin{aligned}
\dot{e}_{i} & =-\lambda_{i} \frac{e_{i}}{\left\|e_{i}\right\|^{1 / 2}}+\left(d_{i}+z_{i}\right) \\
\frac{d\left(d_{i}+z_{i}\right)}{d t} & =-\alpha_{i} \frac{e_{i}}{\left\|e_{i}\right\|}+\dot{d}_{i} .
\end{aligned}
$$

Define $z_{i+1}=d_{i}+z_{i}, i=V, \Omega$, so (37) can be rewritten as follows:

$$
\begin{aligned}
\dot{e}_{i} & =-\lambda_{i} \frac{e_{i}}{\left\|e_{i}\right\|^{1 / 2}}+z_{i+1} \\
\dot{z}_{i+1} & =-\alpha_{i} \frac{e_{i}}{\left\|e_{i}\right\|}+\dot{d}_{i} .
\end{aligned}
$$

Considering (38), we can derive that Theorem 6 is the special case of Theorem 2 when (22) turns into $\left\{\begin{array}{l}\left\|\rho_{1}\right\|=0 \\ \left\|\rho_{2}\right\| \leq \delta_{2}\end{array}\right.$. Thus, the parameters in (23) will turn into the following form:

$$
\begin{aligned}
& \lambda>\sqrt{2 \delta_{2}} \\
& \alpha>3 \delta_{2}+\frac{2 \delta_{2}^{2}}{\lambda^{2}} .
\end{aligned}
$$

3.2.2. Altitude Subsystem. Considering the altitude subsystem dynamics described in (18), the altitude controller can be designed as follows.

Step 1. Define the altitude tracking error as follows:

$$
e_{z}=z_{d}-z
$$

where $z_{d}$ is the reference altitude trajectory, and define the vertical velocity tracking error as follows:

$$
e_{w}=w_{d}-w
$$

Taking the time derivative of (40), it yields

$$
\dot{e}_{z}=\dot{z}_{d}-w .
$$

Substituting (41) into (42), the open-loop altitude tracking error dynamics can be written as follows:

$$
\dot{e}_{z}=\dot{z}_{d}-\left(w_{d}-e_{w}\right),
$$

where $w_{d}$ is the desired vertical velocity. It can be viewed as virtual control and is designed as follows:

$$
w_{d}=\dot{z}_{d}+k_{z} e_{z}
$$

where $k_{z}$ is a positive control gain. Then the closed-loop altitude tracking error dynamics will be obtained as follows:

$$
\dot{e}_{z}=-k_{z} e_{z}+e_{w}
$$

Step 2. Taking the time derivative of (41), the open-loop vertical velocity tracking error dynamics can be written as follows:

$$
\dot{e}_{w}=\dot{w}_{d}-g-r_{33}\left(-g+Z_{w} w+Z_{\mathrm{col}} \delta_{\mathrm{col}}\right)-d_{w} .
$$

Define the collective pitch $\delta_{\text {col }}$ as follows:

$$
\delta_{\text {col }}=\delta_{\text {col_equ }}+\delta_{\text {col_smc }} \text {, }
$$

where $\delta_{\text {col_equ }}$ and $\delta_{\text {col_smc }}$ are equivalent control and switching control, respectively.

The equivalent control $\delta_{\text {col_equ }}$ is chosen as follows:

$$
\delta_{\text {col_equ }}=\frac{\left(\dot{w}_{d}-g-r_{33}\left(-g+Z_{w} w\right)-\widehat{d}_{w}\right)}{r_{33} Z_{\text {col }}},
$$

where $\widehat{d}_{w}$ is the estimated disturbance in altitude subsystem.

Substituting (47) and (48) into (46), we can obtain the dynamics of $e_{w}(t)$ as follows:

$$
\dot{e}_{w}=-r_{33} Z_{\text {col }} \delta_{\text {col_smc }}-\tilde{d}_{w},
$$

where $\widetilde{d}_{w}=d_{w}-\widehat{d}_{w}$ is the disturbance estimation error.

The sliding variable can be chosen as follows:

$$
s_{w}=e_{w}
$$

3.2.3. Yaw Subsystem. Considering the yaw subsystem dynamics described in (19), the design procedure of the yaw controller is similar to the altitude controller in Section 3.2.2. Thus, the simplified procedure is given as follows.

Step 3. The open-loop yaw tracking error dynamics can be written as follows:

$$
\dot{e}_{\psi}=\dot{\psi}_{d}-\left(\frac{S_{\phi}}{C_{\theta}} q+\frac{C_{\phi}}{C_{\theta}}\left(r_{d}-e_{r}\right)\right)
$$

where $r_{d}$ is the virtual control designed as follows:

$$
r_{d}=\frac{C_{\theta}}{C_{\phi}}\left(\dot{\psi}_{d}-\frac{S_{\phi}}{C_{\theta}} q+k_{\psi} e_{\psi}\right),
$$

where $k_{\psi}$ is a positive control gain. Then the closed-loop yaw tracking error dynamics will be obtained as follows:

$$
\dot{e}_{\psi}=-k_{\psi} e_{\psi}+\frac{C_{\phi}}{C_{\theta}} e_{r} .
$$

Step 4. The open-loop yaw angular velocity error dynamics can be written as follows:

$$
\begin{aligned}
\dot{e}_{r}= & \dot{r}_{d}+\frac{I_{y y}-I_{x x}}{I_{z z}} p q-N_{r} r-N_{\mathrm{col}} \delta_{\mathrm{col}}-N_{\mathrm{ped}} \delta_{\mathrm{ped}} \\
& -d_{r} .
\end{aligned}
$$

Define the collective pitch of the tail rotor $\delta_{\text {ped }}$ as follows:

$$
\delta_{\text {ped }}=\delta_{\text {ped_equ }}+\delta_{\text {ped_smc }} \text {. }
$$


The equivalent control $\delta_{\text {ped_equ }}$ is chosen as follows:

$$
\begin{aligned}
& \delta_{\text {ped_equ }} \\
& =\frac{\left(\dot{r}_{d}+\left(\left(I_{y y}-I_{x x}\right) / I_{z z}\right) p q-N_{r} r-N_{\text {col }} \delta_{\text {col }}-\widehat{d}_{r}\right)}{N_{\text {ped }}},
\end{aligned}
$$

where $\widehat{d}_{r}$ is the estimated disturbance in yaw subsystem.

Substituting (55) and (56) into (54), we can obtain the dynamics of $e_{r}(t)$ as follows:

$$
\dot{e}_{r}=-N_{\text {ped }} \delta_{\text {ped_smc }}-\tilde{d}_{r},
$$

where $\widetilde{d}_{r}=d_{r}-\widehat{d}_{r}$ is the disturbance estimation error.

The sliding variable can be chosen as follows:

$$
s_{r}=e_{r} .
$$

3.2.4. Horizontal Subsystem. Considering the horizontal subsystem dynamics described in (20), the horizontal controller can be designed as follows.

Step 5. Define the horizontal tracking error as follows:

$$
e_{P 1}=P_{1 d}-P_{1} \text {, }
$$

where $P_{1 d}=\left[\begin{array}{ll}x_{d} & y_{d}\end{array}\right]^{T}$ is the reference horizontal trajectory, and define the horizontal velocity tracking error as follows:

$$
e_{V 1}=V_{1 d}-V_{1}
$$

Taking the time derivative of (59), it yields

$$
\dot{e}_{P 1}=\dot{P}_{1 d}-V_{1}
$$

Substituting (60) into (61), the open-loop horizontal tracking error dynamics can be written as follows:

$$
\dot{e}_{P 1}=\dot{P}_{1 d}-\left(V_{1 d}-e_{V 1}\right) \text {. }
$$

We design the virtual control $V_{1 d}$ as follows:

$$
V_{1 d}=\dot{P}_{1 d}+k_{P 1} e_{P 1}
$$

where $k_{P 1}=\operatorname{diag}\left(k_{P 11}, k_{P 12}\right)$ is a diagonal matrix of positive control gain. Then the closed-loop horizontal trajectory tracking error dynamics will be obtained as follows:

$$
\dot{e}_{P 1}=-k_{P 1} e_{P 1}+e_{V 1} .
$$

Step 6. Taking the time derivative of (60), the open-loop horizontal velocity tracking error dynamics can be written as follows:

$$
\dot{e}_{V 1}=\dot{V}_{1 d}-R_{1}\left(-g+Z_{w} w+Z_{\mathrm{col}} \delta_{\mathrm{col}}\right)-d_{V 1} \text {. }
$$

The orientation error is defined as follows:

$$
e_{R 1}=R_{1 d}-R_{1} \text {. }
$$

Substituting (66) into (65), the open-loop horizontal velocity tracking error dynamics can be rewritten as follows:

$$
\begin{aligned}
\dot{e}_{V 1}= & \dot{V}_{1 d}-\left(R_{1 d}-e_{R 1}\right)\left(-g+Z_{w} w+Z_{\mathrm{col}} \delta_{\mathrm{col}}\right) \\
& -d_{V 1} .
\end{aligned}
$$

The desired orientation $R_{1 d}$ is viewed as virtual control and can be chosen as follows:

$$
R_{1 d}=\frac{\dot{V}_{1 d}-\widehat{d}_{V 1}+k_{V 1} e_{V 1}+e_{P 1}}{-g+Z_{w} w+Z_{\mathrm{col}} \delta_{\mathrm{col}}},
$$

where $k_{V 1}=\operatorname{diag}\left(k_{V 11}, k_{V 12}\right)$ is a diagonal matrix of positive control gain, and $\widehat{d}_{V 1}$ is the estimated disturbance in horizontal subsystem. Then the closed-loop horizontal velocity tracking error dynamics will be obtained as follows:

$$
\begin{aligned}
\dot{e}_{V 1}= & -k_{V 1} e_{V 1}-e_{P 1}-\tilde{d}_{V 1} \\
& +e_{R 1}\left(-g+Z_{w} w+Z_{\mathrm{col}} \delta_{\mathrm{col}}\right),
\end{aligned}
$$

where $\widetilde{d}_{V 1}=d_{V 1}-\widehat{d}_{V 1}$ is the disturbance estimation error.

Step 7. Taking the time derivative of (66), the open-loop orientation error dynamics can be written as follows:

$$
\dot{e}_{R 1}=\dot{R}_{1 d}-\Pi_{1} \Omega_{1} .
$$

We define the horizontal angular velocity error as follows:

$$
e_{\Omega 1}=\Omega_{1 d}-\Omega_{1} \text {. }
$$

Substituting (71) into (70), the open-loop orientation error dynamics can be rewritten as follows:

$$
\dot{e}_{R 1}=\dot{R}_{1 d}-\Pi_{1}\left(\Omega_{1 d}-e_{\Omega 1}\right) .
$$

The virtual control $\Omega_{1 d}$ can be designed as follows:

$$
\begin{aligned}
& \Omega_{1 d} \\
& \quad=\Pi_{1}^{-1}\left(\dot{R}_{1 d}+e_{V 1}\left(-g+Z_{w} w+Z_{\mathrm{col}} \delta_{\mathrm{col}}\right)+k_{R 1} e_{R 1}\right),
\end{aligned}
$$

where $k_{R 1}=\operatorname{diag}\left(k_{R 11}, k_{R 12}\right)$ is a diagonal matrix of positive control gain. Then the closed-loop orientation error dynamics will be obtained as follows:

$$
\dot{e}_{R 1}=-k_{R 1} e_{R 1}+\Pi_{1} e_{\Omega 1}-e_{V 1}\left(-g+Z_{w} w+Z_{\mathrm{col}} \delta_{\mathrm{col}}\right) .
$$

Step 8. Taking the time derivative of (71), the open-loop horizontal angular velocity error dynamics can be written as follows:

$$
\dot{e}_{\Omega 1}=\dot{\Omega}_{1 d}-f_{1}\left(\Omega_{1}\right)-A_{1} \Omega_{1}-B_{1} u_{1}-d_{\Omega 1} .
$$

The control law $u_{1}$ is designed as follows:

$$
u_{1}=u_{1 \_ \text {equ }}+u_{1 \_ \text {smc }} \text {, }
$$

where $u_{1 \_ \text {equ }}$ and $u_{1 \_ \text {smc }}$ are equivalent control and switching control, respectively. 
The equivalent control $u_{1 \_ \text {equ }}$ is chosen as follows:

$$
u_{1 \_ \text {equ }}=B_{1}^{-1}\left(\dot{\Omega}_{1 d}-f_{1}\left(\Omega_{1}\right)-A_{1} \Omega_{1}-\widehat{d}_{\Omega 1}\right) \text {, }
$$

where $\widehat{d}_{\Omega 1}$ is the estimated disturbance in horizontal subsystem.

Substituting (76) and (77) into (75), we can obtain the dynamics of $e_{\Omega 1}(t)$ as follows:

$$
\dot{e}_{\Omega 1}=-B_{1} u_{1 \_s m c}-\tilde{d}_{\Omega 1},
$$

where $\widetilde{d}_{\Omega 1}=d_{\Omega 1}-\widehat{d}_{\Omega 1}$ is the disturbance estimation error.

The sliding variable can be chosen as follows:

$$
s_{\Omega 1}=e_{\Omega 1} \text {. }
$$

3.2.5. Multivariable Super Twisting Control Law. We rewrite (49), (57), and (78) as the following form:

$$
\begin{gathered}
\dot{s}_{w}=-r_{33} Z_{\text {col }} \delta_{\text {col_smc }}-\tilde{d}_{w}=v_{w}-\widetilde{d}_{w} \\
\dot{s}_{r}=-N_{\text {ped }} \delta_{\text {ped_smc }}-\tilde{d}_{r}=v_{r}-\tilde{d}_{r} \\
\dot{s}_{\Omega 1}=-B_{1} u_{1 \_s m c}-\tilde{d}_{\Omega 1}=v_{\Omega 1}-\tilde{d}_{\Omega 1} .
\end{gathered}
$$

These three sliding mode systems can be summarized to a multivariable canonical form:

$$
\dot{s}_{i}=v_{i}+\widetilde{d}_{i},
$$

where $\widetilde{d}_{i}=d_{i}-\widehat{d}_{i} \in R^{m \times 1}$ is the disturbance estimation error, and $s_{i}, v_{i} \in R^{m \times 1}$ are the sliding variable and control input vector, respectively.

From Theorem 6 , we can obtain that $\widetilde{d}_{i}$ will converge to zero in finite time. Thus, it will be reasonable to state the following assumption.

Assumption 7. The disturbance estimation error $\widetilde{d}_{i}$ is globally bounded by

$$
\left\|\tilde{d}_{i}\right\|<a_{i}\left\|s_{i}\right\|^{1 / 2}, \quad i=V, \Omega
$$

for some constants $a_{i}>0$.

The multivariable super twisting control law can be designed as follows:

$$
\begin{aligned}
& v_{i}=-\lambda_{i} \frac{s_{i}}{\left\|s_{i}\right\|^{1 / 2}}+z_{i} \\
& \dot{z}_{i}=-\alpha_{i} \frac{s_{i}}{\left\|s_{i}\right\|},
\end{aligned}
$$

where $\lambda_{i}, \alpha_{i}$ are controller gains.

Theorem 8. Consider system (83) under Assumption 7. If the parameters in control law (85) satisfy

$$
\begin{aligned}
& \lambda_{i}>2 a_{i}, \\
& \alpha_{i}>\lambda_{i} \frac{4 a_{i} \lambda_{i}+2.25 a_{i}^{2}}{2\left(\lambda_{i}-2 a_{i}\right)},
\end{aligned}
$$

all the trajectories of system (83) converge to the origin $s_{i}=0$ in finite time.
Proof. Considering (83) and (84), we can derive that Theorem 8 also is the special case of Theorem 2 when (22) turns into $\left\{\begin{array}{c}\left\|\rho_{1}\right\| \leq \delta_{1}\left\|x_{1}\right\|^{1 / 2} \\ \left\|\rho_{2}\right\|=0\end{array}\right.$. Thus, the controller parameters in (23) will turn into the following form:

$$
\begin{aligned}
& \lambda>2 \delta_{1} \\
& \alpha>\lambda \frac{4 \lambda+2.25 \delta_{1}^{2}}{2\left(\lambda-2 \delta_{1}\right)} .
\end{aligned}
$$

From (80)-(86), we can obtain the control signals $v_{w}, v_{r}$, and $v_{\Omega 1}$. Thus, the switching control inputs $\delta_{\text {col_smc }}, \delta_{\text {ped_smc' }}$, $\delta_{\text {lat_smc }}$, and $\delta_{\text {lon_smc }}$ also can be derived. Combining (48), (56), and (77), the control input vector $u=\left[\begin{array}{llll}\delta_{\text {col }} & \delta_{\text {lon }} & \delta_{\text {lat }} & \delta_{\text {ped }}\end{array}\right]^{T}$ will be derived as follows:

$$
u=u_{\text {equ }}+u_{\text {smc }}
$$

Remark 9. Systems (80) and (81) are both the special cases of multivariable system (83), where the dimension is $m=1$; that is to say, the control signals $v_{w}$ and $v_{r}$ are the single-variable super twisting control laws.

Remark 10. The discontinuities in control law (85) will only occur when all the components of the vector $s_{i}$ equal zero, but for decoupled single-variable super twisting algorithm, the discontinuities will occur when any of the components of the vector $s_{i}$ equals zero. And the observers (31) and (32) have the same characteristic too. Therefore, the proposed nondecoupled multivariable super twisting algorithm has improved chattering reduction properties relative to decoupled singlevariable super twisting algorithm in some degree.

3.2.6. Super Twisting Based Differentiator. In the controller design procedure, some derivatives of virtual controls are required. In order to lessen calculative burden, super twisting algorithm based exact differentiators are deigned as follows:

$$
\begin{aligned}
& \dot{\hat{x}}=-k_{1 \_i}|\widehat{x}-x|^{1 / 2} \operatorname{sign}(\widehat{x}-x)+\widehat{v} \\
& \dot{\hat{v}}=-k_{2 \_i} \operatorname{sign}(\widehat{x}-x),
\end{aligned}
$$

where $\widehat{x}$ is the estimate of $x$, and $\widehat{v}$ is the estimate of $\dot{x}$. When the parameters $k_{1}$ and $k_{2}$ are chosen appropriately, $\hat{v}$ will converge to $\dot{x}$ in finite time. So $\widehat{v}$ will be used in the controller instead of $\dot{x}$.

Among these virtual controls, the derivatives of (68) and (73) are quite complicated, so these two derivatives will be estimated by exact differentiators.

\section{Stability Analysis for Unmanned Helicopter System}

In this section, we will give the stability analysis for unmanned helicopter system by Lyapunov technique. 
The three closed-loop subsystems are displayed as follows:

$$
\begin{aligned}
& S_{1}:\left\{\begin{array}{l}
\dot{e}_{z}=-k_{z} e_{z}+s_{w} \\
\dot{s}_{w}=v_{w}-\widetilde{d}_{w}
\end{array}\right. \\
& S_{2}:\left\{\begin{array}{l}
\dot{e}_{\psi}=-k_{\psi} e_{\psi}+\frac{C_{\phi}}{C_{\theta}} s_{r} \\
\dot{s}_{r}=v_{r}-\widetilde{d}_{r}
\end{array}\right. \\
& S_{3}: \begin{array}{l}
\dot{e}_{P 1}=-k_{P 1} e_{P 1}+e_{V 1} \\
\dot{e}_{V 1}=-k_{V 1} e_{V 1}-e_{P 1}-\tilde{d}_{V 1}+e_{R 1}\left(-g+Z_{w} w+Z_{\mathrm{col}} \delta_{\mathrm{col}}\right) \\
\dot{e}_{R 1}=-k_{R 1} e_{R 1}-e_{V 1}\left(-g+Z_{w} w+Z_{\mathrm{col}} \delta_{\mathrm{col}}\right)+\Pi_{1} s_{\Omega 1} \\
\dot{s}_{\Omega 1}=v_{\Omega 1}-\tilde{d}_{\Omega 1},
\end{array}
\end{aligned}
$$

where $v_{w}, v_{r}$, and $v_{\Omega 1}$ represent super twisting control laws, and $\widetilde{d}_{w}, \widetilde{d}_{r}, \widetilde{d}_{V 1}$, and $\widetilde{d}_{\Omega 1}$ represent disturbance estimation errors.

Theorem 11. Consider the helicopter model (18)-(20), under Assumptions 1-7. If the disturbance observers are designed as (31) and (32), and the control inputs are chosen as (88), the position and yaw tracking errors globally asymptotic converge to zero.

Proof. From the above analysis, we can conclude that sliding mode variable $s_{i}$ will converge to manifold $s_{i}=0$ in finite time and remain zero for all subsequent time, and disturbance estimation error $\tilde{d}_{i}$ will converge to zero in finite time too. Therefore, the closed-loop system dynamics are governed by the following reduced order system:

$$
\begin{aligned}
& S_{1}: \dot{e}_{z}=-k_{z} e_{z} \\
& S_{2}: \dot{e}_{\psi}=-k_{\psi} e_{\psi} \\
& S_{3}: \\
& \qquad \begin{array}{l}
\dot{e}_{P 1}=-k_{P 1} e_{P 1}+e_{V 1} \\
\dot{e}_{V 1}=-k_{V 1} e_{V 1}-e_{P 1}+e_{R 1}\left(-g+Z_{w} w+Z_{\mathrm{col}} \delta_{\mathrm{col}}\right) \\
\dot{e}_{R 1}=-k_{R 1} e_{R 1}-e_{V 1}\left(-g+Z_{w} w+Z_{\mathrm{col}} \delta_{\mathrm{col}}\right) .
\end{array}
\end{aligned}
$$

\begin{tabular}{|c|c|c|c|c|c|c|}
\hline Variable & Description & \multicolumn{5}{|c|}{ Value } \\
\hline$m$ & $\begin{array}{l}\text { Mass of } \\
\text { unmanned } \\
\text { helicopter }\end{array}$ & \multicolumn{5}{|c|}{$8.2 \mathrm{~kg}$} \\
\hline$g$ & $\begin{array}{l}\text { Acceleration of } \\
\text { gravity }\end{array}$ & \multicolumn{5}{|c|}{$9.81 \mathrm{~m} \cdot \mathrm{s}^{-2}$} \\
\hline$Z_{w}$ & $\begin{array}{l}\text { Linkage gain } \\
\text { ratio of } T \text { to } w\end{array}$ & \multicolumn{5}{|c|}{$-0.7615 \mathrm{~s}^{-1}$} \\
\hline$Z_{\mathrm{col}}$ & $\begin{array}{l}\text { Linkage gain } \\
\text { ratio of } T \text { to } \delta_{\text {col }}\end{array}$ & \multicolumn{5}{|c|}{$-131.4125 \mathrm{~m} /\left(\mathrm{rad} \cdot \mathrm{s}^{2}\right)$} \\
\hline$J$ & $\begin{array}{l}\text { The moment of } \\
\text { inertia }\end{array}$ & \multicolumn{5}{|c|}{$\operatorname{diag}\{0.18,0.34,0.28\} \mathrm{kg} \cdot \mathrm{m}^{2}$} \\
\hline$A$ & $\begin{array}{l}\text { Coefficient } \\
\text { matrix of } \Omega \text { in } \\
(9)\end{array}$ & \multicolumn{5}{|c|}{$\operatorname{diag}\{-48.1757,-25.5048,-0.9808\} \mathrm{s}^{-1}$} \\
\hline$B$ & $\begin{array}{l}\text { Coefficient } \\
\text { matrix of } u \text { in } \\
\text { (9) }\end{array}$ & {$\left[\begin{array}{c}0 \\
0 \\
-0.3705\end{array}\right.$} & $\begin{array}{c}0 \\
894.5 \\
0\end{array}$ & $\begin{array}{c}1689.5 \\
0 \\
0\end{array}$ & $\left.\begin{array}{c}0 \\
0 \\
135.8\end{array}\right]$ & $\mathrm{s}^{-2}$ \\
\hline
\end{tabular}

Considering the above closed-loop system, we define a Lyapunov function candidate as follows:

$$
V(t)=V_{1}(t)+V_{2}(t)+V_{3}(t)
$$

where $V_{1}(t), V_{2}(t)$, and $V_{3}(t)$ can be defined, respectively, as

$$
\begin{aligned}
& V_{1}(t)=\frac{1}{2} e_{z}^{T} e_{z} \\
& V_{2}(t)=\frac{1}{2} e_{\psi}^{T} e_{\psi} \\
& V_{3}(t)=\frac{1}{2} e_{P 1}^{T} e_{P 1}+\frac{1}{2} e_{V 1}^{T} e_{V 1}+\frac{1}{2} e_{R 1}^{T} e_{R 1} .
\end{aligned}
$$

TABLE 1: Parameters of unmanned helicopter.

By taking the time derivation of (92), we can derive the following equation:

$$
\begin{aligned}
\dot{V}( & (t)=e_{z}^{T} \dot{e}_{z}+e_{\psi}^{T} \dot{e}_{\psi}+e_{P 1}^{T} \dot{e}_{P 1}+e_{V 1}^{T} \dot{e}_{V 1}+e_{R 1}^{T} \dot{e}_{R 1} \\
& =e_{z}^{T}\left(-k_{z} e_{z}\right)+e_{\psi}^{T}\left(-k_{\psi} e_{\psi}\right)+e_{P 1}^{T}\left(-k_{P 1} e_{P 1}+e_{V 1}\right) \\
& +e_{V 1}^{T}\left(-k_{V 1} e_{V 1}-e_{P 1}\right. \\
& \left.+e_{R 1}\left(-g+Z_{w} w+Z_{\mathrm{col}} \delta_{\mathrm{col}}\right)\right)+e_{R 1}^{T}\left(-k_{R 1} e_{R 1}\right. \\
& \left.-e_{V 1}\left(-g+Z_{w} w+Z_{\mathrm{col}} \delta_{\mathrm{col}}\right)\right)=-k_{z} e_{z}^{T} e_{z} \\
& -k_{\psi} e_{\psi}^{T} e_{\psi}-e_{P 1}^{T} k_{P 1} e_{P 1}-e_{V 1}^{T} k_{V 1} e_{V 1}-e_{R 1}^{T} k_{R 1} e_{R 1} \\
& <0
\end{aligned}
$$

To sum up, the position and yaw tracking errors will globally asymptotically converge to zero.

\section{Simulation Results and Discussion}

In order to verify the effectiveness of the proposed control scheme to the unmanned helicopter, simulation tests are presented in this section. The numerical simulations are conducted in the MATLAB. The parameters of unmanned helicopter are shown in Table 1, and the parameters of the proposed controller are shown in Table 2.

The proposed control method will be compared with the control method proposed in [24] to demonstrate the merits of it. Moreover, the control method proposed in [24] is backstepping sliding mode control (BSMC) method without disturbance observer.

The slalom flight path is a typical trajectory to examine the maneuver performance. It can test the tracking accuracy, 
TABLE 2: Parameters of controller.

\begin{tabular}{lcc}
\hline Variable & Value & Description \\
\hline$K_{Z}$ & 1 & Control gain in (44) \\
$K_{\Psi}$ & 5 & Control gain in (52) \\
$K_{P 1}$ & $\operatorname{diag}\{1,1\}$ & Control gain in (63) \\
$K_{V 1}$ & $\operatorname{diag}\{3,3\}$ & Control gain in (68) \\
$K_{R 1}$ & $\operatorname{diag}\{5,5\}$ & Control gain in (73) \\
$\lambda_{w}$ & 0.15 & Control gain for (80) \\
$\alpha_{w}$ & 0.05 & Control gain for (80) \\
$\lambda_{r}$ & 0.5 & Control gain for (81) \\
$\alpha_{r}$ & 1.5 & Control gain for (81) \\
$\lambda_{\Omega 1}$ & 0.5 & Control gain for (82) \\
$\alpha_{\Omega 1}$ & 1.5 & Control gain for (82) \\
$\lambda_{V}$ & 1 & Observer gain in (31) \\
$\alpha_{V}$ & 3 & Observer gain in (31) \\
$\lambda_{\Omega}$ & 2 & Observer gain in (32) \\
$\alpha_{\Omega}$ & 10 & Observer gain in (32) \\
$k_{1 \_68}$ & 20 & Differentiator gain for (68) \\
$k_{2 \_68}$ & 30 & Differentiator gain for (68) \\
$k_{1 \_73}$ & 20 & Differentiator gain for (73) \\
$k_{2 \_73}$ & 30 & Differentiator gain for (73) \\
\hline
\end{tabular}
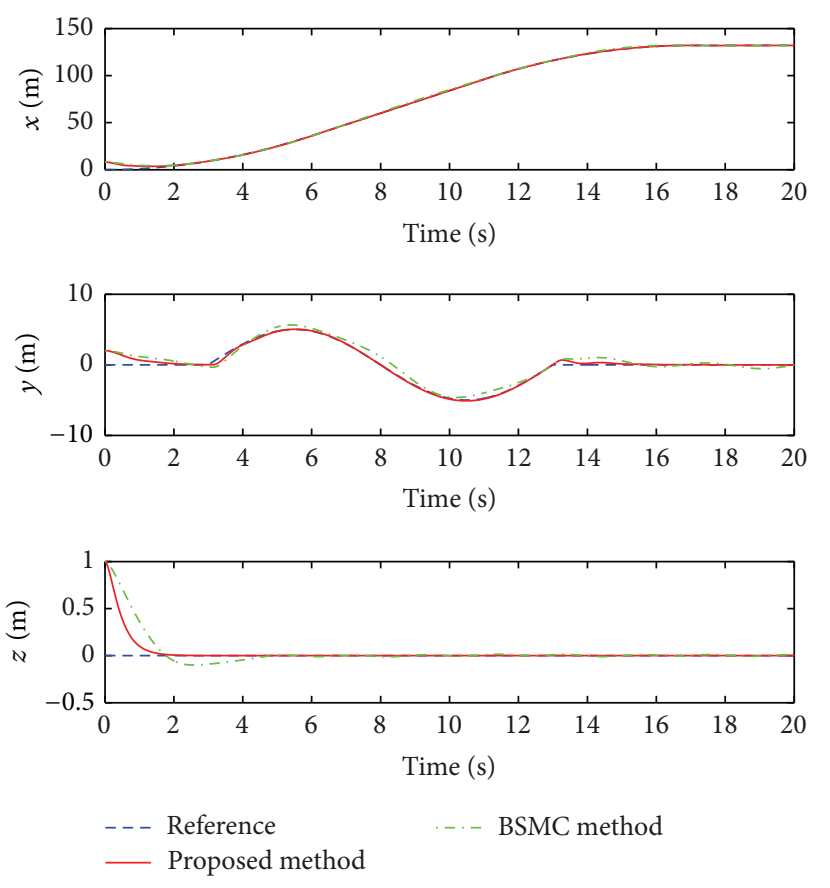

FIgURE 3: Position tracking responses.

robustness, and coupling properties. The desired slalom flight path $P_{d}=\left[\begin{array}{lll}x_{d} & y_{d} & z_{d}\end{array}\right]^{T}$ and $\psi_{d}$ are designed as follows:

$$
x_{d}(t)= \begin{cases}t^{2} & t \leq 6 \mathrm{~s} \\ 12 t-36 & 6<t \leq 11 \mathrm{~s} \\ -t^{2}+34 t-157 & 11<t \leq 17 \mathrm{~s} \\ 132 & t>17 \mathrm{~s}\end{cases}
$$
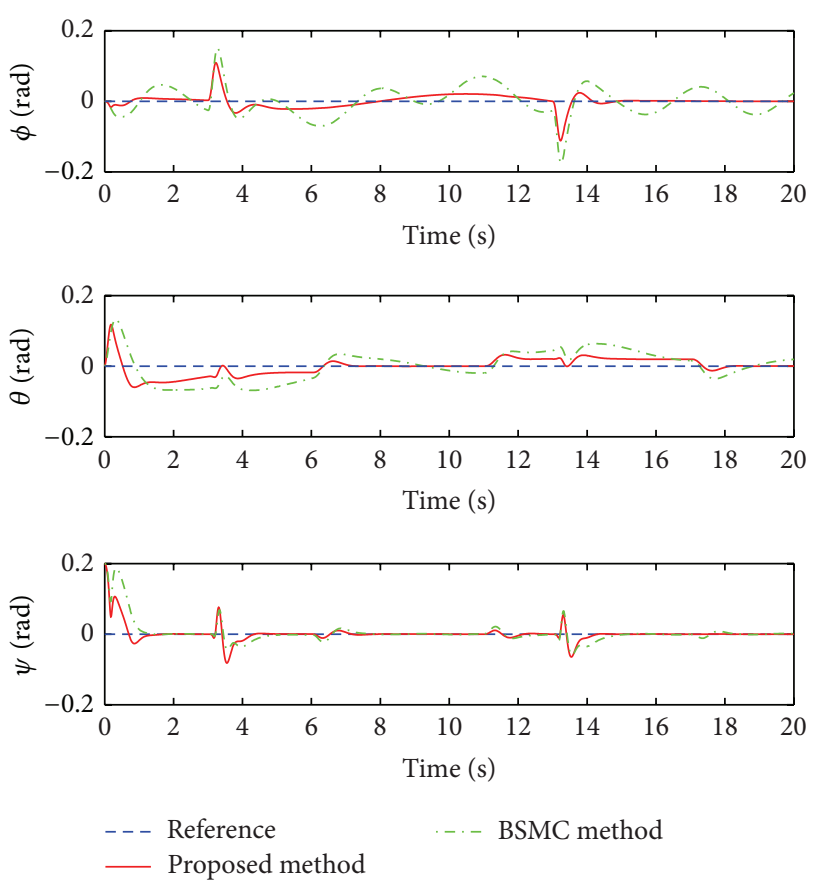

Figure 4: Euler angle tracking responses.

$$
\begin{aligned}
& y_{d}(t)= \begin{cases}0 & t \leq 3 \mathrm{~s} \\
5 \sin \left(\frac{\pi}{5}(t-3)\right) & 3<t \leq 13 \mathrm{~s} \\
0 & t>13 \mathrm{~s}\end{cases} \\
& z_{d}(t)=0 \\
& \psi_{d}(t)=0 .
\end{aligned}
$$

In order to demonstrate the excellent tracking performance comprehensively, nonzero initial position and Euler angle are set as $P_{0}=\left[\begin{array}{lll}8 & 2 & 1\end{array}\right]^{T} m$ and $\Theta_{0}=\left[\begin{array}{lll}0 & 0 & 0.2\end{array}\right]^{T} \mathrm{rad}$.

During the simulation, the model uncertainties and external disturbances $d=\Delta\left[\begin{array}{llll}P^{T} & V^{T} & \Theta^{T} & \Omega^{T}\end{array}\right]^{T}+d_{\text {wind }}$ are added to verify the robustness of the proposed controller, where $\Delta \in R^{6 \times 12}$ represents the model uncertainty matrix, and all of its elements are pseudorandom values on the interval $(-0.1,0.1)$. The external disturbance is $d_{\text {wind }}=$ $\left[\begin{array}{llll}\sin (0.1 t) & 2 \sin (0.1 t) & 0.5 \sin (0.1 t) & 0_{1 \times 3}\end{array}\right]^{T}$.

The simulation results are illustrated in Figures 36. Figure 3 shows the position tracking responses, while Figure 4 depicts the Euler angle tracking responses. Through these figures, one can see that the proposed controller is superior to the BSMC in [24] for its tracking performance, which is specific in its gentler dynamic process, faster tracking ability, and smaller overshoot. Moreover, the control inputs of the proposed method are smoother than the BSMC proposed in [24] which suffer from chattering phenomena seriously (see Figure 5). Finally, Figure 6 illustrates the effectiveness of the designed disturbance observer. One can see that 

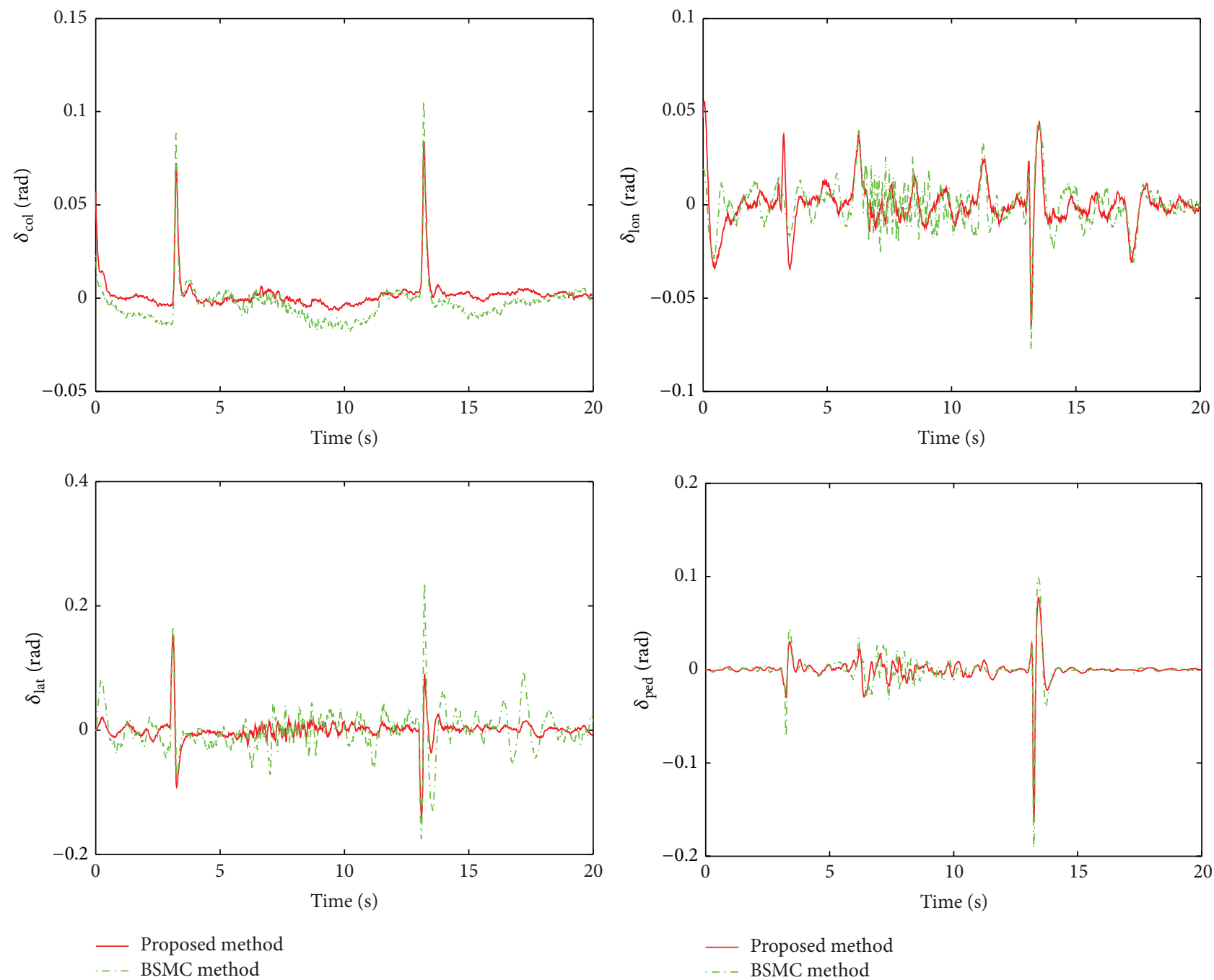

FIGURE 5: Control inputs.

the observer can estimate the compounded disturbances accurately.

From the simulation results, we can obtain that the proposed controller is very effective in controlling the unmanned helicopter in the presence of both matched and unmatched disturbances. However, some limitations of the controller should be discussed here. First, the proposed control scheme is a hierarchical control strategy. The controller structure is somewhat complex, so we need to design it very carefully. Second, there are still many parameters to be known in the helicopter model and controller.

\section{Conclusion}

A disturbance observer based controller using backstepping and multivariable super twisting control technique is developed for small unmanned helicopters. The compounded disturbances and the time derivatives of the virtual controls are estimated by super twisting based observer and differentiator. The proposed controller possesses the robustness with both matched and unmatched disturbances. The finite time stability of the modified multivariable super twisting algorithm and global asymptotic stability of the closed-loop helicopter system are proved by Lyapunov theory. Finally, numerical simulation results demonstrate the effectiveness of the proposed flight control scheme, which is superior to the BSMC in [24] for its accurate tracking performance and chattering attenuation ability. The proposed control method will be tested on the experimental helicopter platform in future work.

\section{Conflict of Interests}

The authors declare that there is no conflict of interests regarding the publication of this paper. 

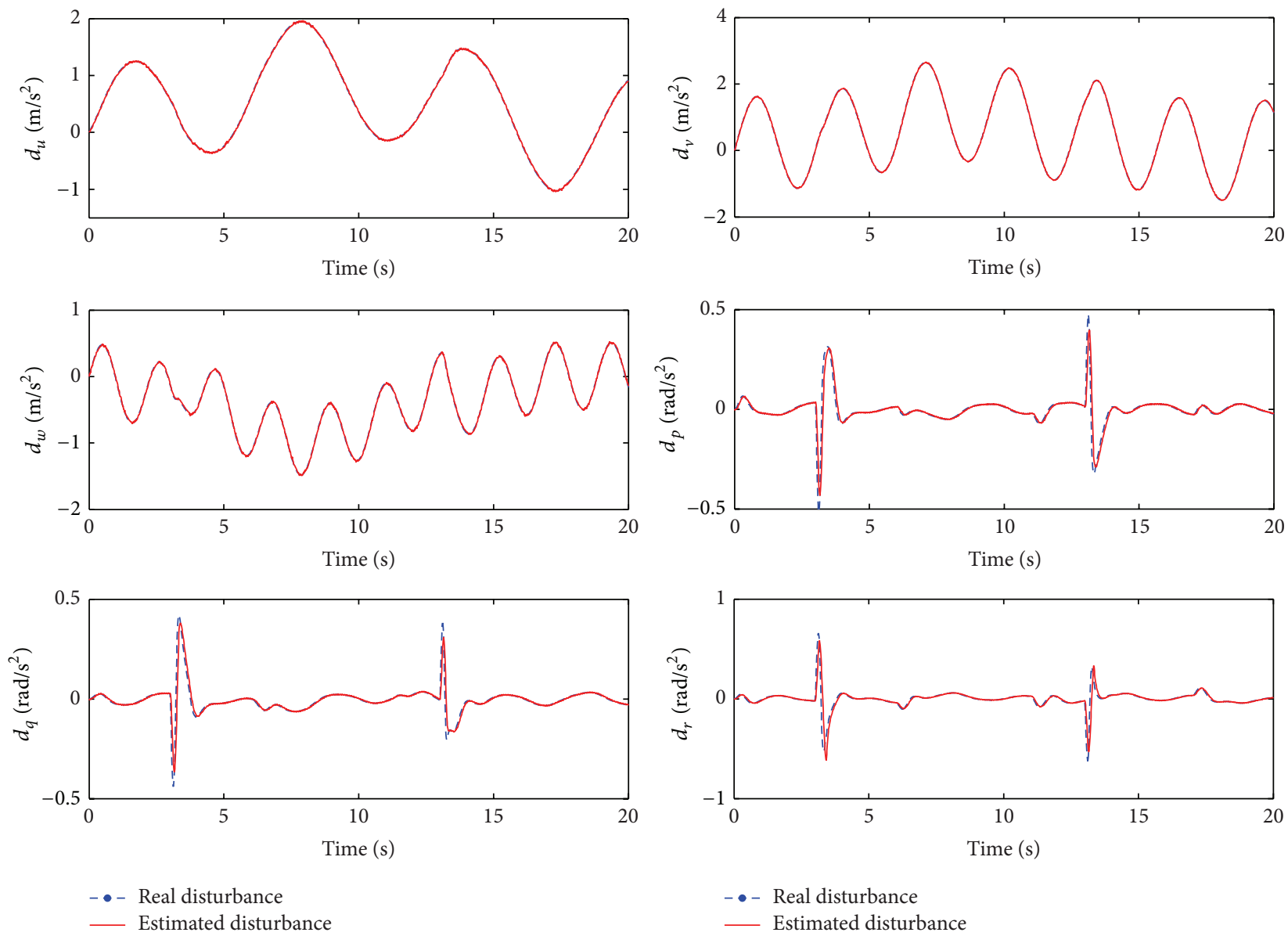

FIGURE 6: Estimated disturbances.

\section{Acknowledgments}

This work is supported by the Natural Science Foundation of China (61403274) and the Application Base and Frontier Technology Research Project of Tianjin of China (13JCQNJC03600).

\section{References}

[1] J. Shin, K. Nonami, and D. Fujiwara, "Model-based optimal attitude and positioning control of small-scale unmanned helicopter," Robotica, vol. 23, no. 1, pp. 51-63, 2005.

[2] G. W. Cai, B. M. Chen, and T. H. Lee, Unmanned Rotorcraft Systems, Springer, New York, NY, USA, 2011.

[3] S. Bouabdallah, A. Noth, and R. Siegwart, "PID vs LQ control techniques applied to an indoor micro Quadrotor," in Proceedings of the IEEE/RSJ International Conference on Intelligent Robots and Systems (IROS '04), pp. 2451-2456, October 2004.

[4] J. J. Gribble, "Linear quadratic Gaussian/loop transfer recovery design for a helicopter in low-speed flight," Journal of Guidance, Control, and Dynamics, vol. 16, no. 4, pp. 754-761, 1993.

[5] J. Gadewadikar, F. L. Lewis, K. Subbarao, and B. M. Chen, "Structured $H_{\infty}$ command and control-loop design for unmanned helicopters," Journal of Guidance, Control, and Dynamics, vol. 31, no. 4, pp. 1093-1102, 2008.
[6] J. Gadewadikar, F. L. Lewis, K. Subbarao, K. Peng, and B. M. Chen, "H-infinity static output-feedback control for rotorcraft," Journal of Intelligent and Robotic Systems: Theory and Applications, vol. 54, no. 4, pp. 629-646, 2009.

[7] D. Maclay and S. J. Williams, "Use of mu-synthesis in full envelope, helicopter flight control system design," in Proceedings of the IEEE International Conference on Control, pp. 718-723, March 1991.

[8] N. Kim, A. J. Calise, N. Hovakimyan, J. V. R. Prasad, and E. Corban, "Adaptive output feedback for high-bandwidth flight control," Journal of Guidance, Control, and Dynamics, vol. 25, no. 6, pp. 993-1002, 2002.

[9] C. Liu, W.-H. Chen, and J. Andrews, "Tracking control of smallscale helicopters using explicit nonlinear MPC augmented with disturbance observers," Control Engineering Practice, vol. 20, no. 3, pp. 258-268, 2012.

[10] M. Chen, S. S. Ge, and B. Ren, "Robust attitude control of helicopters with actuator dynamics using neural networks," IET Control Theory \& Applications, vol. 4, no. 12, pp. 2837-2854, 2010.

[11] Y.-B. He, H.-L. Pei, H.-B. Zhou, and T.-R. Sun, "Adaptive backstepping-based robust tracking control of unmanned helicopters with disturbances," Control Theory and Applications, vol. 30, no. 7, pp. 834-843, 2013.

[12] C.-T. Lee and C.-C. Tsai, "Adaptive backstepping integral control of a small-scale helicopter for airdrop missions," Asian Journal of Control, vol. 12, no. 4, pp. 531-541, 2010. 
[13] Z. Fang, W. N. Gao, and L. Zhang, "Robust adaptive integral backstepping control of a 3-DOF helicopter," International Journal of Advanced Robotic Systems, vol. 9, article 79, 8 pages, 2012.

[14] I. A. Raptis, K. P. Valavanis, and W. A. Moreno, "A novel nonlinear backstepping controller design for helicopters using the rotation matrix," IEEE Transactions on Control Systems Technology, vol. 19, no. 2, pp. 465-473, 2011.

[15] J.-J. Xiong and E.-H. Zheng, "Position and attitude tracking control for a quadrotor UAV," ISA Transactions, vol. 53, no. 3 , pp. 725-731, 2014.

[16] L. Besnard, Y. B. Shtessel, and B. Landrum, "Quadrotor vehicle control via sliding mode controller driven by sliding mode disturbance observer," Journal of the Franklin Institute, vol. 349, no. 2, pp. 658-684, 2012.

[17] L. Derafa, L. Fridman, A. Benallegue, and A. Ouldali, "Super twisting control algorithm for the four rotors helicopter attitude tracking problem," in Proceedings of the 11th International Workshop on Variable Structure Systems (VSS '10), pp. 62-67, June 2010.

[18] J. A. Moreno and M. Osorio, "A Lyapunov approach to secondorder sliding mode controllers and observers," in Proceedings of the 47th IEEE Conference on Decision and Control (CDC '08), pp. 2856-2861, December 2008.

[19] J. Picó, E. Picó-Marco, A. Vignoni, and H. de Battista, "Stability preserving maps for finite-time convergence: super-twisting sliding-mode algorithm," Automatica, vol. 49, no. 2, pp. 534539,2013

[20] J. Davila, L. Fridman, and A. Levant, "Second-order slidingmode observer for mechanical systems," IEEE Transactions on Automatic Control, vol. 50, no. 11, pp. 1785-1789, 2005.

[21] S.-B. Wang, X.-M. Wang, R. Xie, and C.-C. Yao, "Robust backstepping control based on disturbance observer for hypersonic vehicle," Control and Decision, vol. 28, no. 10, pp. 1507-1512, 2013.

[22] A. Levant, "Robust exact differentiation via sliding mode technique," Automatica, vol. 34, no. 3, pp. 379-384, 1998.

[23] I. Nagesh and C. Edwards, "A multivariable super-twisting sliding mode approach," Automatica, vol. 50, no. 3, pp. 984-988, 2014.

[24] T. Madani and A. Benallegue, "Backstepping sliding mode control applied to a miniature quadrotor flying robot," in Proceedings of the 32nd Annual Conference on IEEE Industrial Electronics (IECON '06), pp. 700-705, Paris, France, November 2006. 


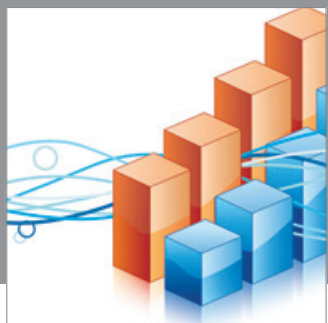

Advances in

Operations Research

mansans

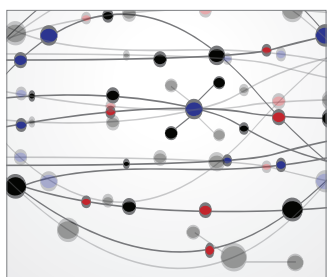

The Scientific World Journal
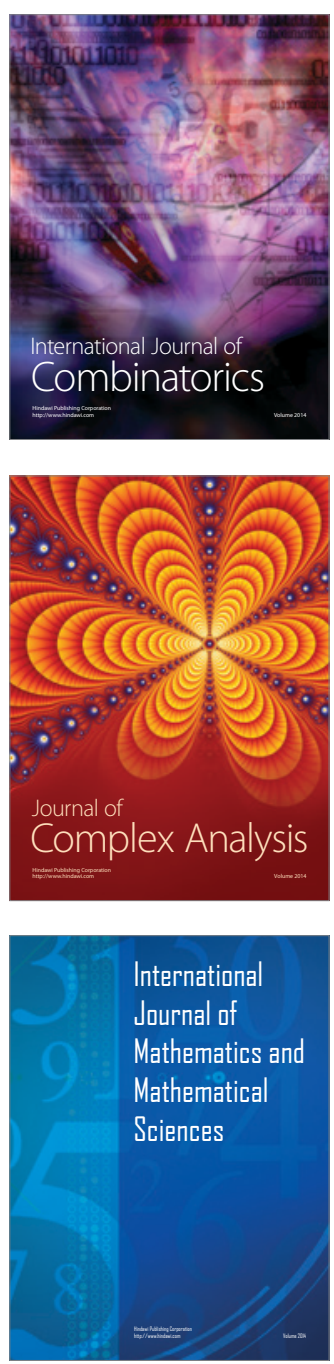
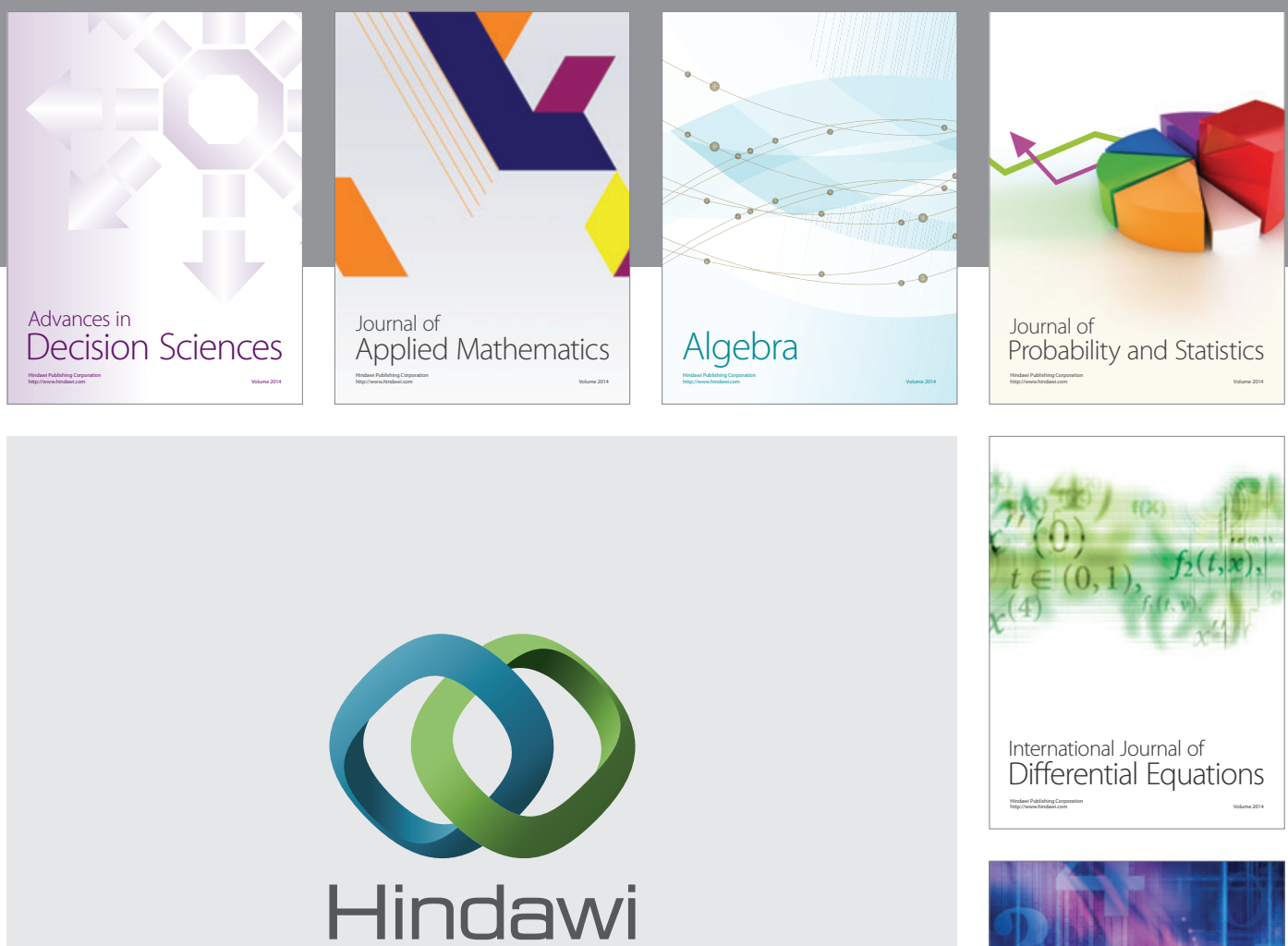

Submit your manuscripts at http://www.hindawi.com
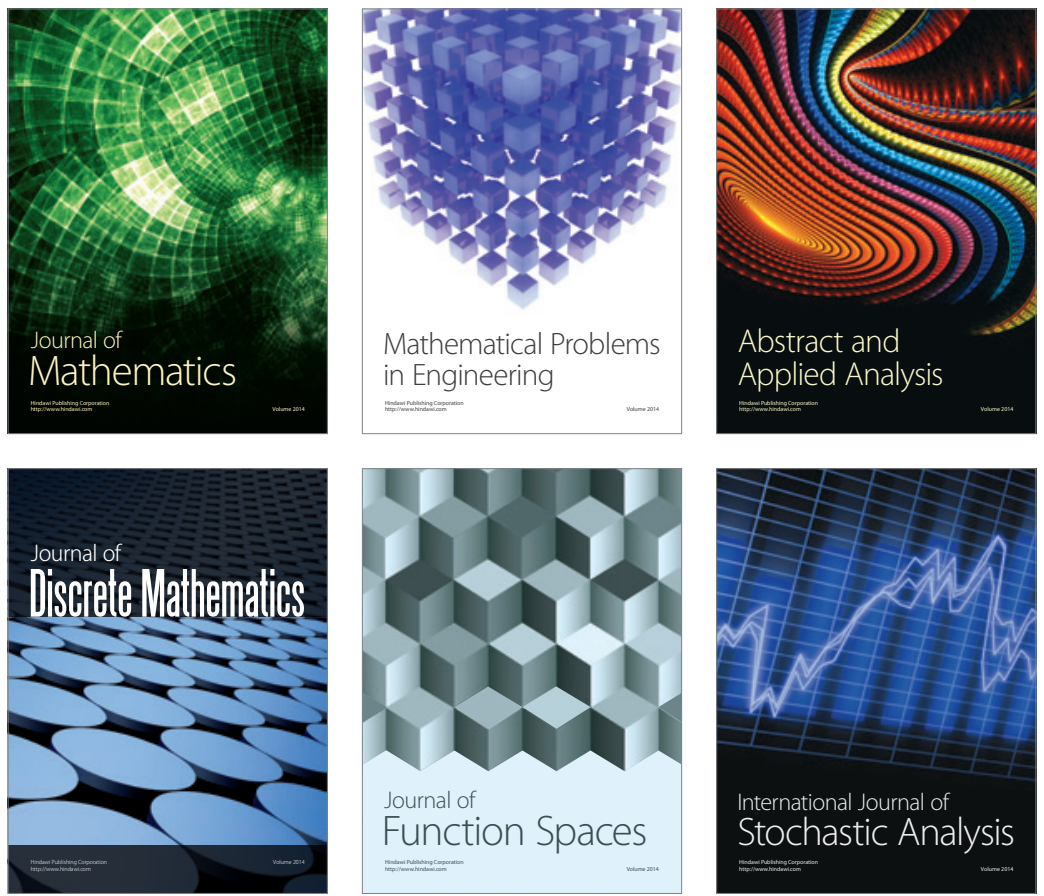

Journal of

Function Spaces

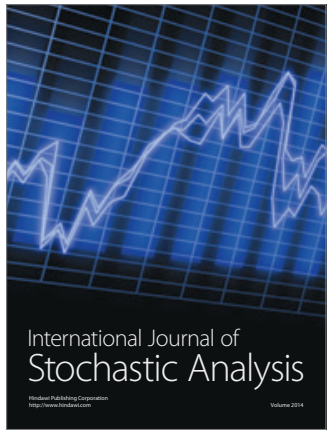

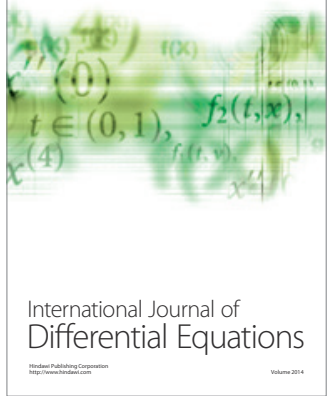
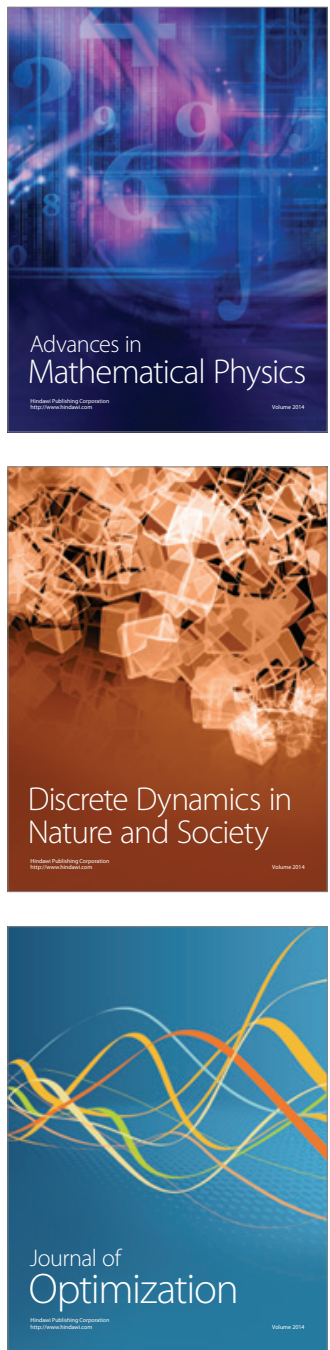